\title{
Group singing as a resource for the development of a healthy public: a study of adult group singing
}

David A. Camlin (1) ${ }^{1 凶}$, Helena Daffern (10 ${ }^{2} \&$ Katherine Zeserson ${ }^{3}$

A growing body of evidence points to a wide range of benefits arising from participation in group singing. Group singing requires participants to engage with each other in a simultaneous musical dialogue in a pluralistic and emergent context, creating a coherent cultural expression through the reflexive negotiation of (musical) meaning manifest in the collective power of the human voice. As such, group singing might be taken-both literally and figuratively-as a potent form of 'healthy public', creating an 'ideal' community, which participants can subsequently mobilise as a positive resource for everyday life. The experiences of a group of singers $(n=78)$ who had participated in an outdoor singing project were collected and analysed using a three-layer research design consisting of: distributed data generation and interpretation, considered against comparative data from other singing groups $(n=88)$; a focus group workshop $(n=11)$; an unstructured interview $(n=2)$. The study confirmed an expected perception of the social bonding effect of group singing, highlighting affordances for interpersonal attunement and attachment alongside a powerful individual sense of feeling 'uplifted'. This study presents a novel perspective on group singing, highlighting the importance of participant experience as a means of understanding music as a holistic and complex adaptive system. It validates findings about group singing from previous studies-in particular the stability of the social bonding effect as a less variant characteristic in the face of environmental and other situational influences, alongside its capacity for mental health recovery. It establishes a subjective sociocultural and musical understanding of group singing, by expanding on these findings to centralise the importance of individual experience, and the consciousness of that experience as descriptive and reflective self-awareness. The ways in which participants describe and discuss their experiences of group singing and its benefits points to a complex interdependence between a number of musical, neurobiological and psychosocial mechanisms, which might be independently and objectively analysed. An emerging theory is that at least some of the potency of group singing is as a resource where people can rehearse and perform 'healthy' relationships, further emphasising its potential as a resource for healthy publics.

\footnotetext{
${ }^{1}$ Royal College of Music, London, UK. ${ }^{2}$ York Centre for Singing Science, AudioLab, University of York, York, UK. ${ }^{3}$ Harmony Associates, Newcastle-uponTyne, UK. ${ }^{凶}$ email: dave.camlin@rcm.ac.uk
} 


\section{Introduction}

significant body of evidence points to the positive health and wellbeing benefits associated with group singing (Skingley and Bungay 2010; Clift 2013; Coulton et al. 2015; Fancourt et al. 2016; Williams et al. 2018; Dingle et al. 2019), which have broad appeal to a wide public (Eno 2008; Burkeman 2015). This article contributes to such discussion by further illustrating the importance of representing participants' personal perspectives of group singing (Dingle et al. 2019, p. 10) through an analysis of their experiences. This approach enhances a more scientific understanding of the natural processes involved, illuminating the complex ways in which group singing might produce its benefits, and highlighting some of its essential characteristics.

While 'participation in singing groups confers significant benefits in terms of mental aspects of quality of life' (Coulton et al. 2015) a detailed understanding of these complex benefits are still emerging. The recent NICE report on group singing and older people suggests: 'the committee... noted that it is unclear whether it is the singing itself that produces the benefit, the group-based nature of the activity or something else (NICE 2015, p. 23).

Perhaps the reason this 'something else' eludes comprehension is because the benefits of group singing consist of a set of interdependencies that might be thought of as an 'adaptive complex', i.e., where 'the functional significance of each characteristic is amplified by the others' (Whiten 2007, p. 155). A comprehensive understanding of any single dimension of the wellbeing effect of group singing is confounded by its many other variable dimensions, and controlling for these variables becomes harder to do as the increasing complexity of their interdependence emerges. Focusing on participant experience provides a way of illuminating this complex interdependence, helping to ground scientific knowledge in the complex reality of people's lived experiences.

Music might be thought of as a polyvalent system, in that its meaning and value can be expressed in a diverse set of complementary dimensions. Even more passive experiences such as musical listening 'occur in a complex interplay between the listener, the music, and the context' (Västfjäll et al. 2013, p. 408), while more complex forms of 'musicking' (Small 1998)-such as participating in and performing music-activate a wide range of interconnected effects, benefits, meanings and significances.

Some of these 'paramusical' (Stige et al. 2013, p. 298) effects include: music's impact on our sense of self and our social and personal identity (DeNora 2000); its capacity for producing or facilitating 'strong' experiences (Gabrielsson 2011); its eudaimonic effect, or positive impact on our health and wellbeing (Ryan and Deci 2001; Bailey and Davidson 2005; Livesey et al. 2012; Creech 2014; Shakespeare and Whieldon 2017); and its capacity to facilitate trust and cooperation, social cohesion, community formation and transformation (Buglass and Webster 2004; Bailey and Davidson 2005; Belfiore and Bennett 2008; Turino 2008; Livesey et al. 2012; DeNora 2013; Hallam 2015; Bartleet 2016; Elliott et al. 2016; Bartleet and Higgins 2018). These effects might be seen to arise from within two broadly complementary musical traditions of performance and participation, i.e., the aesthetic traditions of the public performance of musical works on the one hand, and participation in musical activities on the other. A number of writers (Elliott 1995; Small 1998; Turino 2008; Elliott and Silverman 2013, 2014) have consistently advanced a philosophy of music, which recognises that this binary characterisation of music is misleading and simplistic. The complex network of benefits and effects might more rightly be said to arise across a continuum of creative tension between these two musical dimensions, emphasising the unifying character of the 'paramusical' as a 'third' dimension (Camlin
2014, 2016), which is pertinent in the formation of healthy publics. $^{2}$

A feature of all human society, group singing is perhaps the most accessible form of music-making, and undertaken as a very diverse set of practices across all cultures, from the entirely participatory traditions found within everyday 'social life' (Turino 2008) through to the highly presentational forms of the concert hall. Previous studies have highlighted the potential of group singing as a cost-effective activity with benefits that can be accessed by a broad population, including those in recovery of their mental health (Clift and Morrison 2011; Coulton et al. 2015; Clift et al. 2017; Dingle et al. 2017; Shakespeare and Whieldon 2017; Williams et al. 2018). In relation to the treatment of Persons With Dementia (PWDs), singing is already identified as a means of 'promoting the wellbeing of family members' as well as the PWDs themselves, at least in the medium term (Särkämö et al. 2013, p. 648), suggesting its value as a form of 'mutual recovery' (Crawford et al. 2013, 2015, p. 149).

The benefits of group singing can be expressed in terms of both individual and interpersonal effects, underpinned by musical, neurobiological, communicative, and cultural interdependences.

Individual effects. Listening to and performing music generally modulates levels of a variety of hormones (Levitin et al. 2017, p. 1 ), with group singing particularly implicated (Fancourt et al. 2016, p. 2), especially in the production of oxytocin (Macdonald and Macdonald 2010, pp. 12-14). The production of endorphins also provides a neurohormonal underpinning of the social bonding effect during group musical activities because of the synchronous exchanges which group musical activities facilitate (Tarr et al. 2014, pp. 1-2). There are of course challenges in isolating the effect of any one of these neurohormones, because of their co-variance within musical experience (Tarr et al. 2014, p. 5).

There is a similar growing body of evidence for the individual benefits of group singing in terms of wellbeing (Clift 2013; Clift et al. 2017; Shakespeare and Whieldon 2017; Dingle et al. 2019), emotion regulation (Dingle et al. 2017) and physical health, particularly in relation to respiratory function and treatment of Parkinson's Disease (BBC Health Check 2018; Kreutz et al. 2003, 2004; Skingley et al. 2011; Clift et al. 2013; Morrison and Clift 2013), again with similar challenges relating to isolating particular effects because of the polyvalent complexity of group singing as an activity.

Interpersonal effects. Much of the research into group singingand music more generally-emphasises its social dimension (Livesey et al. 2012; Welch et al. 2014), even though a medical understanding of wellbeing has tended to focus on the impact on the individual, rather than the group. What lies at the heart of musical activity is the co-construction of a social reality through music, binding each individual to the other individuals, to the whole group, and often with those listening. Cultural anthropology has an established understanding of the relational aspects of performance and art making as a core ritual in community building, community thinking and the rehearsal of social roles, where 'musical performance is a part of its social setting' (Chernoff 1979, p. 153), as a manifestation of shared social and cultural values, and where 'people express their opinions by participating' (p.153). An interpersonal understanding of group singing is therefore a way of enhancing current intra-personal knowledge with a broader perspective that accounts for this cocultural phenomenon. 
As the NICE (2015) report implies, because social isolation and loneliness are established indicators of a heightened risk of premature mortality (Holt-Lunstad et al. 2015, p. 247), at least part of the wellbeing effect of group singing might arise because it is a primarily social activity. The potential of group singing as a resource for healthy publics can therefore be made on the basis of its interpersonal affordances. Being socially active can in itself be 'an important predictor of health outcomes' (Dingle et al. 2013, p. 3) and (von Lob et al. 2010) highlight the important relationship between inter-personal and intra-personal mechanisms implicated in group singing in psychosocial terms. This understanding of the social mechanisms that underpin the eudaimonic potential of group singing can be enhanced by an additional consideration of both the musical and neurobiological mechanisms, which may also be implicated, with recent studies pointing to the complex ways in which music and neurobiology entangle themselves (Vickhoff et al. 2013).

Musical entrainment. At the heart of an understanding of the power of music as a mediator of social experience is the phenomenon of 'entrainment' (Clayton 2012). Musical entrainment is 'a phenomenon in which two or more independent rhythmic processes synchronise with each other' (Clayton et al. 2004, p. 1). As entrainment entails 'the shared synchronisation of internal oscillators' (p. 99) it might also extend to other musical features such as the synchronisation of vibrato between singers (Daffern 2017). The stronger the entrainment effect between performers, the more the sound can be collectively controlled to produce particular musical effects (e.g., vocal 'blend'), which can in turn have a positive affect for those participating and/or listening.

Implicated within this synchronisation of musical features between individuals and across groups of musical agents is therefore the collective activation of the mirror neuron system (MNS) (Molnar-Szakacs and Overy 2006, p. 235) as a way of establishing and maintaining such synchronisation. However, rather than a mechanical or metronomic adherence to a fixed pulse, entrainment theory suggests that if two rhythms are entrained, they do not necessarily fall precisely into phase with one another' (Clayton 2012, p. 50). It is precisely the 'participatory discrepancies' that Keil identified as the power of music, whereby music must be out of time and tune to be valuable and involving (Keil 1987, p. 275), which facilitate and inspire participation (Turino 2008, p. 45).

This negotiation of self and other in relation to music, of coming in and out of phase with the musical contributions of the other performers or participants in a 'simultaneous dialogue' (Barenboim 2009, p. 20), leads to what (Tarr et al. 2014) refer to as the phenomenon of 'self-other merging':

'When our own actions match those of another's, it is possible that the intrinsic and extrinsic engagement of neural action-perception networks make it difficult to distinguish between self and perceived other, thus creating at least a transient bond between the two.' (p. 3)

Resonance circuitry. Through these various processes, one can see how music can help to facilitate bonds of trust and attachment, as the resulting synchrony is qualitatively similar to that experienced through similar non-musical bonds of emotional attachment: 'the mammalian nervous system depends... on a system of interactive coordination, wherein steadiness comes from synchronisation with nearby attachment figures. We call this mutually synchronising exchange limbic regulation' (Lewis et al. 2001, p. 84). In other words, the metaphorical relationship implied through musical synchronous exchange can become a proxy for an altogether deeper form of human attachment, which underpins wellbeing.
The notion of interpersonal neurobiological 'attunement' (Siegel 2012, 2016), whereby individuals 'attune' to others is observable through the synchronisation of various neurobiological processes (Siegel D (2015) 'Interview with Dan Siegel (personal communication). A key element of the phenomenon of interpersonal 'resonance' is the recognition of it as a shared experience, which explains why it might contribute positively to the experience not just of social bonding, but also the underlying neurobiological mechanism of the experience of 'love' (Lewis et al. 2001; Dunbar 2013, pp. 16-19; Camlin):

When we attune to others we allow our own internal state to shift, to come to resonate with the inner world of another. This resonance is at the heart of the important sense of 'feeling felt' that emerges in close relationships.

(Siegel 2011, p. 27)

Viewed in this way, one might hypothesise that at least part of the wellbeing effect of group singing arises not just through a synchronisation of participants' neurobiology to produce particular musical entrainment effects, but also the other way round, with the production of those musical effects reinforcing interpersonal attunement and consequent individual wellbeing, a mutually reinforcing and complex adaptive process.

Music as a communicative medium. The complexity of music as a way of being in relationship with others is made still more complex when one considers some of its other communicative functions. A number of theorists have drawn comparisons between music and language as systems of communication (Sloboda 1986; Miell, MacDonald and Hargreaves 2005; Mithen 2007; Malloch and Trevarthen 2010), while (Cross and Woodruff 2009) advance a model of music as a communicative medium, which recognises three complementary dimensions: a motivational-structural dimension, a culturally enactive dimension, and a socio-intentional dimension (p. 8).

When added to the foregoing complexity of the various neurohormonal activations involved, interpersonal and intrapersonal considerations, the musical elements themselves and how the phenomenon of entrainment threads through all of the above, one gets a clear sense of the complex nature of meaning, which underpins musical experience. Isolating and observing any one aspect of music's agency not only proves to be exceedingly challenging, it also highlights how easy it would be to de-nature the whole phenomenon under investigation, by removing or controlling for dimensions, which in the natural course of things may be co-constituents in the production of meaning.

Methodological justification. To consider group singing as a health-supporting activity in the same way as, for example, diet or exercise, it is important to understand the mechanism/s through which it achieves its effects. However, because of the complex and interdependent nature of these mechanisms, measuring isolated phenomena may be insufficient in revealing the full extent of what is happening during the activity.

Focusing on participant experience-as a way of illuminating some of the already complex meanings within this sympathetic entanglement of multiple dimensions-might be seen as introducing an unnecessary additional level of complexity. However a subjective understanding of experience can provide greater clarity. Rather than attempting to measure and quantify the various inter-relationships implicit in the complex web of meaning described above, the approach taken in this study focuses instead on a combination of analysing the stories participants tell about their experiences, and the sense they make of those experiences through 'self-signification' against a set of 
pre-determined categories. Emphasising how personal and collective 'beliefs, practices, values, and social processes' (Hinchliffe et al. 2018, p. 3) can condition meaning, the method builds understanding of the significance, which individuals attach to their experience of group singing and its assumed benefits.

The approach of comparing participants' narrative accounts does not ignore the complexity surrounding the wellbeing effect of group singing, rather it starts from a position of understanding people's experience of it, and from there looks for any characteristics, which might be more universal. The validity of such insights can be strengthened when correlated with the empirical findings of more scientific studies, as well as illuminating any 'dissonance' between the scientific data and people’s actual experiences.

\section{Methodology}

Addressing the need 'to select a means of data collection and communication that accurately represents both the outcomes/ impacts of arts interventions and the experiences of research subjects' (Skingley et al. 2011), this study used an 'integrative' (Plowright 2010) methodological approach to collect and analyse 'layers' of data arising from a sequence of three interconnected methods:

- Layer 1: Distributed data generation and interpretation $(n=78)$ via Sensemaker ${ }^{\circledast}$ software app

- Layer 2: Focus Group workshop $(n=11)$

- Layer 3: Unstructured interview $(n=2)$

Participants. Participants were recruited from the 'Fellowship of Hill and Wind and Sunshine'3 project (National Trust, 2018), which took four groups of 50-60 amateur singers to sing on Lake District summits. Most singers were involved in regular choirs led by musicians from the vocal group, Mouthful (Mouthful 2020) ${ }^{4}$, within the 'Natural Voice' (Natural Voice Network 2020; Bithell 2014) tradition of aural learning, and they attended singing workshops specific to the Fellowship project. 78 of the Fellowship singers $(51 \mathrm{~F} 27 \mathrm{M})$ participated in the study. A sub-set $(n=11)$ of Fellowship participants $(8 \mathrm{~F}, 3 \mathrm{M})$ participated in the Layer 2 focus group discussion, and $2(2 \mathrm{~F})$ participants with therapeutic professional backgrounds participated in the Layer 3 unstructured interview.

A comparison project collected data from $(n=88)$ singers (67F, 21M) across 24 regular UK community choirs, again with the majority led by musicians from Mouthful and in the 'Natural Voice' style but not combined with a specific outdoor walking project. These data are referred to as non-Fellowship and are used for comparison with the Fellowship data set in Layer 1. Some of these singers may have also been involved in the Fellowship project but due to anonymity these data aren't known and they are treated separately throughout.

Participation in the research was voluntary, and respondents consented to participate as a pre-requisite for participation, retaining the right to withdraw from the project at any time.

\section{Methods}

Distributed data generation/interpretation. In the first layer of research, qualitative data in the form of personal narrative accounts were collected via a process of distributed data generation (Snowden 2016) using the Sensemaker ${ }^{\circledR}$ software app. Involving participants in a process of 'self-signification' (Cognitive Edge 2019) of their experiences in this way-albeit against a series of categories pre-determined by the research team in the research design stage-helped to limit any implicit bias arising from the pre-existing relationship between researcher/s and participants.

Following their singing experience, each respondent used the Sensemaker ${ }^{\Phi}$ software app to choose from one of three 'prompt' questions to stimulate a response in the form of a story 'fragment' or micro-narrative:

i. Tell a story about an experience that influenced your decision to participate/not participate in group singing.

ii. Fast forward to a time in the future where you have to teach a younger person a lesson in the value of group singing. What experience would you share?

iii. You are being interviewed by the local media after singing in a group at a recent event. What would you say?

Participants were encouraged to respond in written form but could also use photographs or audio/video recordings if they felt it was appropriate. Eleven respondents marked their micronarrative as 'private', which excluded its content from any thematic analysis, although their 'self-signification' interpretation was still included in the geometric analysis of data.

Participants 'interpreted' their story after submission by mapping it against a series of 'signifier sets', i.e., placing a mark within a series of triangular ternary graphs representing three equally positive dimensions of the experience (see Fig. 1). The process of plotting their experience against a ternary graph 'helps the user to think in a broader, more holistic context by collapsing three options/descriptors/topics into one indication' (Brown et al. 2017, p. 683), providing 'enough cognitive load to force the person signifying to think about placement,' while avoiding' the more traditional good-bad type scale' (Snowden 2011 p.230). In the face of the multiple ways in which a group singing experience might be seen to produce a range of beneficial outcomes-e.g., personal impact, social impact, functional outcomes (Dingle et al. 2013, p. 8)-the ternary graph provides a means of understanding the relative significance of those different benefits in the personal interpretations of participants, which introduces a methodological rigour to approaching the complexity of personal experience.

Each signifier set represented either ideas in current literature about music and group singing, discussed in 'Introduction' section of this paper, or was 'polymorphic' in the sense that it was part of a core group of 14 such sets, used across the whole of the international 'Making of Meaning' project (Cognitive Edge 2018), which uses Sensemaker ${ }^{\circledR}$ as a methodology ${ }^{5}$.

Figure 1 shows an example of the signification interface on an Apple desktop computer:

Respondents interpreted their stories against six sets of signifiers ${ }^{6}$ :

1. Belonging: my people; me; my place

2. Experience: physical; spiritual; mental

3. Engagement: improvement; achievement; enjoyment

4. Value: aesthetic; paramusical; participatory

5. Environment: the group; the leader; the environment

6. Transformation: perceptual; somatic; environmental

The resulting signification data-large-scale graphical 'fitness landscapes' (Snowden 2011, p. 226) of participants' interpretations of their experience within each signifier set-indicate patterns in the ways that respondents interpret their own narratives, as 'clusters' of emergent meaning. These graphical data were analysed through a broad geometric analysis. The stories themselves were then further analysed using Nvivo software to code and divide the data between the predetermined categories, and also to identify other emerging categories and themes. We expected that the Fellowship singers would attribute greater significance to the effect of the 


\section{In the experience shared:}

I have a stronger sense of who I am

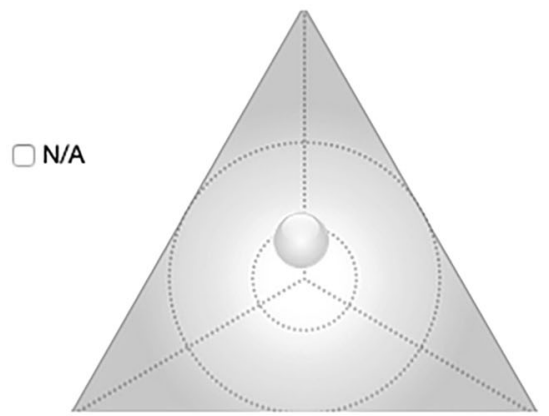

I feel a sense of brother/sisterhood with others in the group connection

\section{In the experience / story I shared, group singing:}

\section{Lifts me out of my everyday experience}

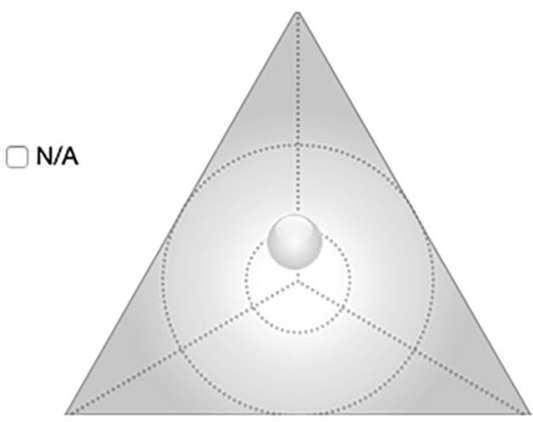

Is good for my physical health

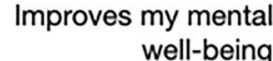
world around me

Fig. 1 Sensemaker ${ }^{\oplus}$ signification interface.

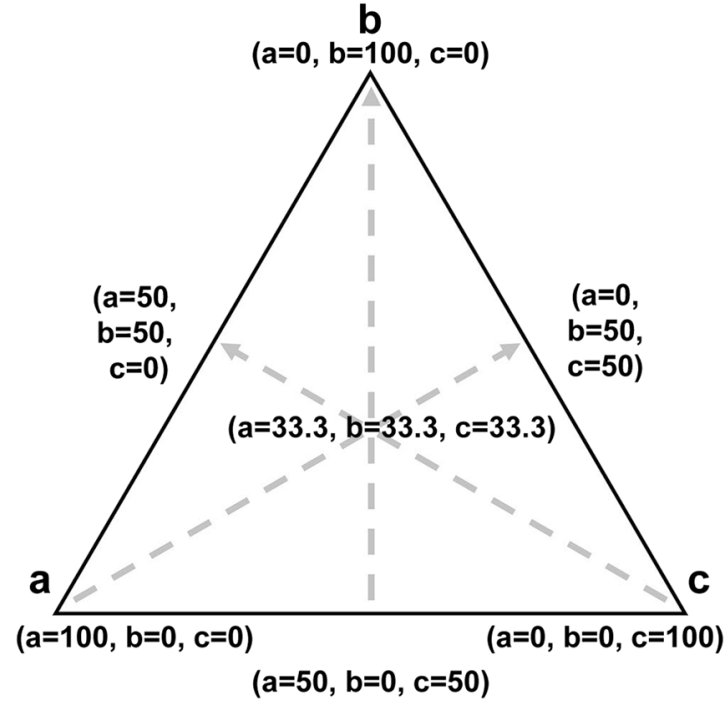

Fig. 2 Distribution of data across three axes within a triangle.

environment on their singing experience compared to the nonfellowship participants, singing under 'normal' conditions. We also expected that the Fellowship singers would attribute greater significance to the considerable physical exertion required to participate in the project.

'Theming' workshop. Following the collection of participants' stories in Layer 1, a focus group of 11 respondents (7F 4M) from the Fellowship project were recruited by open invitation to participate in a 3-h 'theming' workshop, involving them in a process of qualitative analysis of the transcripts and signification data from Layer 1 . The objective here was not only to further draw out emergent themes, but also to facilitate participants' reflective and descriptive self-awareness, thereby increasing the impact of the study on those taking part. Layer 1 'non-private' data (micronarratives and signification data) was circulated to the group beforehand. During the focus group workshop, three groups of three to four people were instructed to discuss the transcripts and the fitness landscapes and to identify what they saw as any emergent or important themes in the data. The groups were instructed to 'share air-time' so that the views of each person in the group had equal representation. They held simultaneous independent discussions without researcher input, before sharing their reflective interpretations with another group of participants, and subsequently participating in a whole group discussion facilitated by one of the researchers, where broad consensus around the emergent themes was agreed.

Focus group interview. To explore emerging themes in more detail, a further unstructured interview was organised between one of the researchers and two participants who had been involved in both previous layers of data collection, and who selfidentified as having professional experience of therapeutic work, as a psychotherapist and a therapeutic social worker. The interview was unstructured, with an initial prompt from the lead researcher to discuss their experiences of both group singing and therapeutic work in relation to the previous discussions in the 'theming' workshop, leading to a full dialogue between them of a range of issues, with occasional prompts from the researcher to re-focus the discussion.

Audio recordings of both the focus group and subsequent interview were made, and a transcript of the discussions thematically analysed using a combination of the categories identified in Layer 1, as well as emergent themes.

\section{Results}

The resulting data were analysed through a combination of geometric analysis of participant 'self-signification' data in Layer 1 alongside thematic analysis of participant narratives and focus group data in Layers 1, 2 and 3. The layers produced rich data, which we were able to cross-reference in the development/validation of our findings.

Geometric analysis of distributed data generation and interpretation. Within the Sensemaker ${ }^{\oplus}$ Analyst software, all of the signifying marks made by respondents within each triangular signifier set during the process of self-signification (above) appear within a single triangle or 'fitness landscape' (Snowden 2011, 


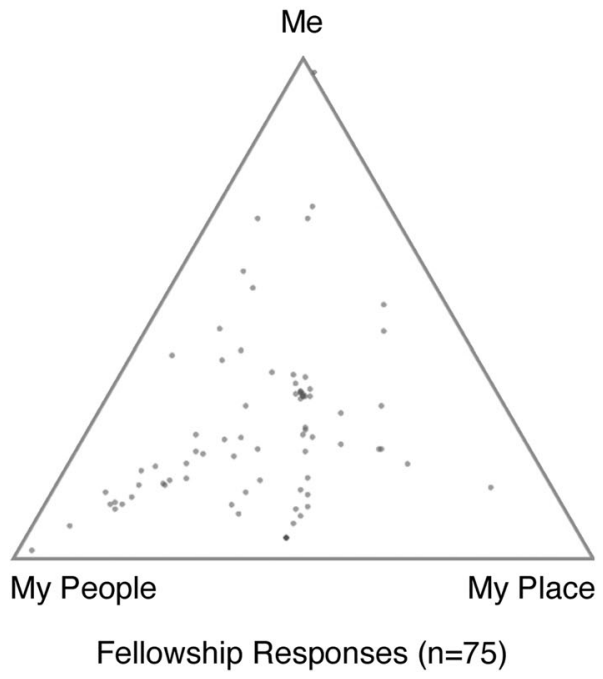

Fig. 3 Responses for Triad 1: Belonging.

p. 226), thus affording a basic comparison of responses across 'three filters or scales, each representing the strength or weakness of one of the labels' (Snowden 2011, p. 231). If one assumes that each axis of the triangle represents a scale of $0-100$, then any mark placed within it will be plotted with reference to these three axes, as shown in Fig. 2:

For example, a mark placed at the very apex of the $b$ axis would return a result of $(a=0, b=100, c=0)$ and the same would be true of the other two axes. A mark placed exactly in the centre of the triangle would return a result of $(a=33.3, b=33.3, c=33.3)$, suggesting an equal emphasis between dimensions. A mark placed elsewhere in the triangle is effectively emphasising either one, or two of the dimensions over the other/s. The closer to the apex of an axis, the higher the corresponding \% along each axis, and the more that axis might be said to be emphasised over the other/s.

A more random distribution of marks would be expected to be evenly distributed across the different dimensions of the triangle, without observable 'clustering'. Hence, the first basic analysis is to visually observe patterns in the distribution of marks within each fitness landscape, especially where individual marks 'cluster' around a particular part of the triangle. In the geometric analysis which follows, it is assumed that any mark that returns a percentage score of 34 or more against any given axis is effectively emphasising it over at least one of the other axes. This does not therefore attempt to directly measure the strength of feeling with which a mark might have been placed against a particular dimension; however, it provides initial insight into the data from where to explore a more detailed thematic comparison of responses.

One of the advantages of using Sensemaker ${ }^{\circledast}$ is that a basic visual analysis of the fitness landscapes arising from the process of self-signification reveals patterns of signification, which appear to be less stable, and hence less worthy of further investigation. Of the six sets of signifier, three (Engagement, Environment, Transformation) produced fitness landscapes with more even patterns of signification, i.e., with responses more evenly spread across the three dimensions. Accordingly, these were excluded from further geometric analysis. The remaining three (Belonging, Experience, Value) revealed more visually observable patterns/ clusters of data, which warranted further investigation.

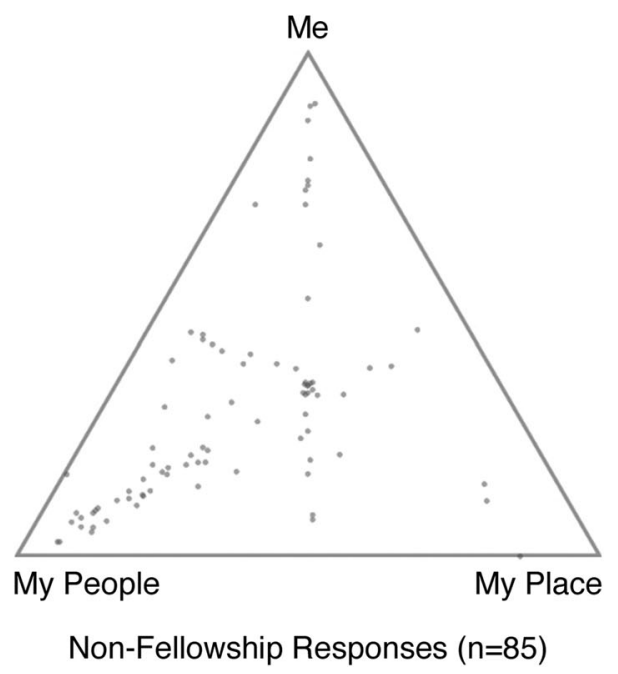

Belonging (Triad 1: My People, Me, My Place). Figure 3 shows how respondents interpreted their experience against the three complementary dimensions of:

- Social/My People (I feel a sense of brother/sisterhood with others in the group);

- Personal/Me (I have a stronger sense of who I am);

- Situational/My Place (I experience a stronger connection to the world around me)

A comparison was made between the Fellowship singers $(n=75)$ and the non-Fellowship singers $(n=85)$, and similar patterns of signification were found:

The number and percentage of responses rated $34 \%$ or above against each dimension:

- My People (social)

- $76 \%$ of Fellowship responses $(n=58)$

- $74 \%$ of non-Fellowship responses $(n=63)$

- $\mathrm{Me}$ (personal)

- $29 \%$ of Fellowship responses $(n=22)$

- $36 \%$ of non-Fellowship responses $(n=31)$

- My Place (situational)

- $42 \%$ of Fellowship responses $(n=32)$

- $25 \%$ of non-Fellowship responses $(n=21)$

The importance of the social dimension (My People) was similar across both groups, while My Place was emphasised more by the Fellowship participants. This confirms somewhat our expectation that the Fellowship singers would emphasise a 'sense of place' more strongly in their interpretation of their experience. However, while they do emphasise such an effect, it was not at the expense of the social impact of the experience.

Experience (Triad 2: Physical, Spiritual, Mental) $(\mathrm{n}=161)$. Respondents interpreted their experience against three complementary dimensions of: 


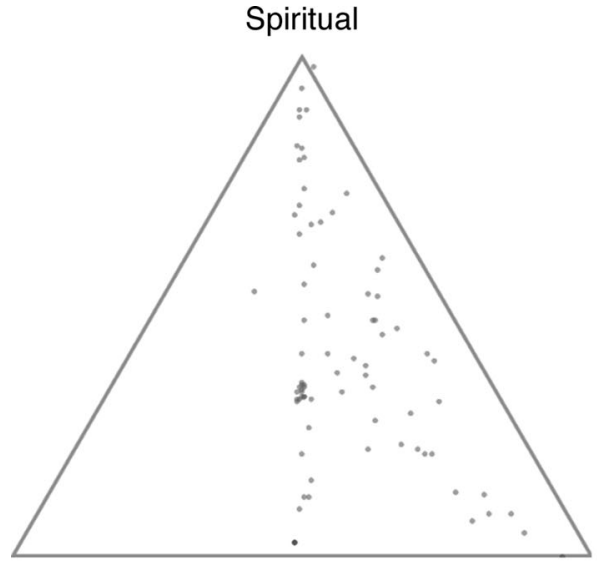

Physical
Psychological

Fellowship Responses $(\mathrm{n}=77)$

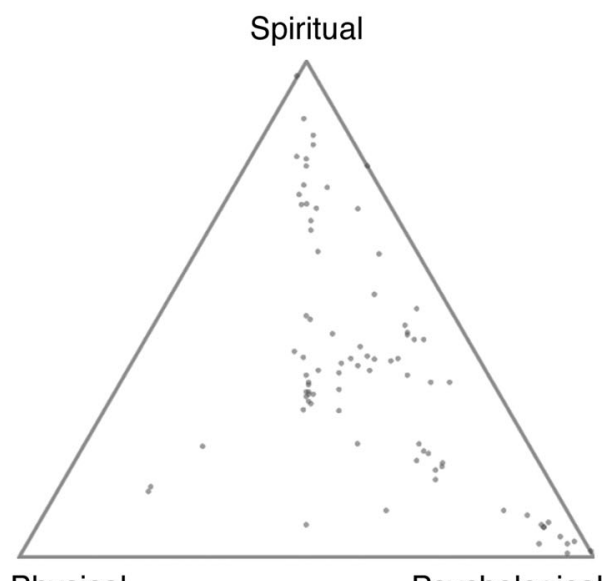

Physical

Non-Fellowship Responses $(n=84)$

- 36\% of Fellowship responses $(n=28)$

- $52 \%$ of non-Fellowship responses $(n=44)$

A high proportion of Fellowship participants emphasised the Paramusical in their interpretation, but, as Fig. 5 shows, very few highlighted Aesthetic over the other dimensions. This contrasts with non-Fellowship responses, which show a more even distribution of emphasis to include the Aesthetic dimension.

Summary of geometric analysis of distributed data generation/ interpretation. In summary, the geometric analysis of the selfsignification data of layer 1 suggests that:

- The social dimension (My People) of group singing is more significant in participants' interpretation than that of the personal (Me) or the situational (My Place), and this appears to be invariant across 'normal' conditions (non-Fellowship data set) and the conditions of an outdoor singing project (Fellowship data set);

- The spiritual/psychological dimensions of group singing are interpreted by participants as more significant than benefits to their physical health, and this appears to be maintained even when the activity involves high levels of physical exertion, i.e., mountain hiking;

- Participants interpret paramusical outcomes and participation as more important than aesthetic concerns, although this interpretation is only apparent in the Fellowship data set.

Thematic analysis of distributed data generation and interpretation. To complement the geometric analysis of participants' self-signification, the narrative data collected through the Sensemaker ${ }^{\circledR}$ process provide a broad overview of the ways in which respondents subjectively interpret their experience, and hence point toward characteristics of the experience, which might be more collectively held. To save space, most of the stories themselves are not included herein, but links to the stories are provided as footnotes. Respondents' micro-narratives were initially coded against the pre-determined categories contained within each fitness landscape. In this initial coding, particular words/ terms/sentence meanings were assigned a certain code, with some flexibility in assignation owing to the richness of the narrative data. Subsequent analysis of the data within Nvivo software organised it into dependent sub-themes. Figure 6 provides an 


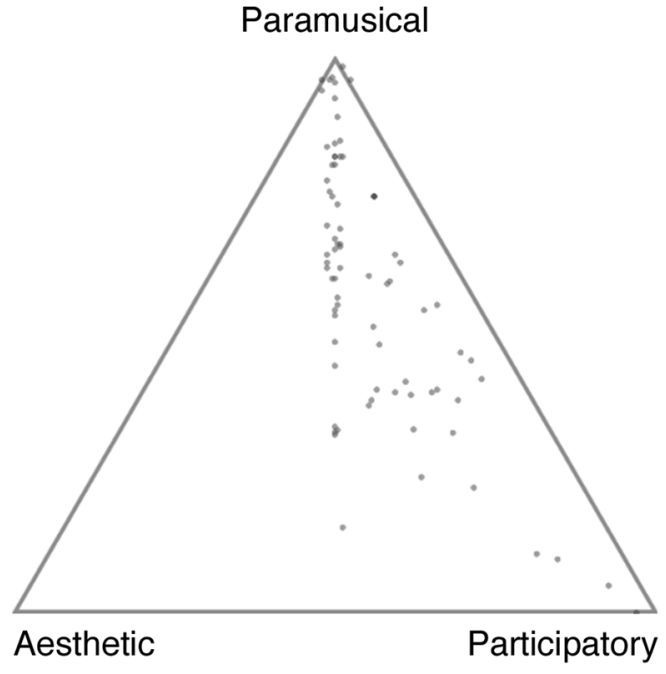

Fellowship Responses ( $\mathrm{n}=76)$

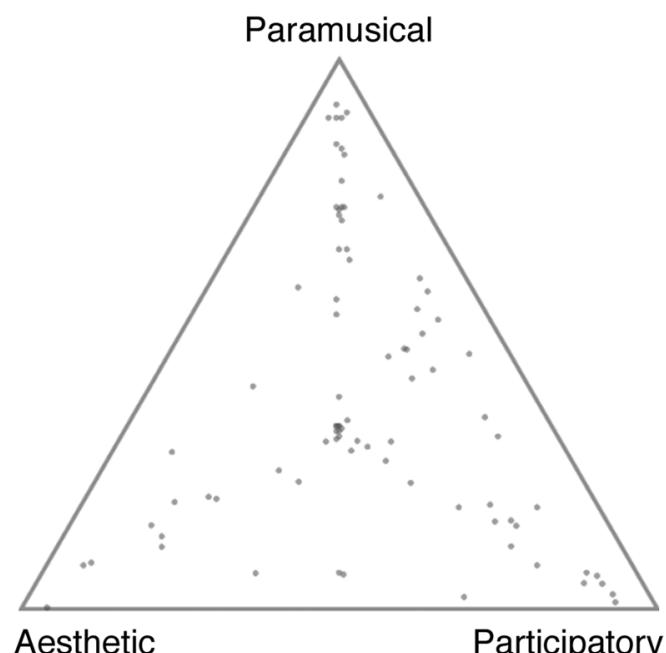

Non-Fellowship Responses $(n=84)$

Fig. 5 Responses for Triad 4: Value.

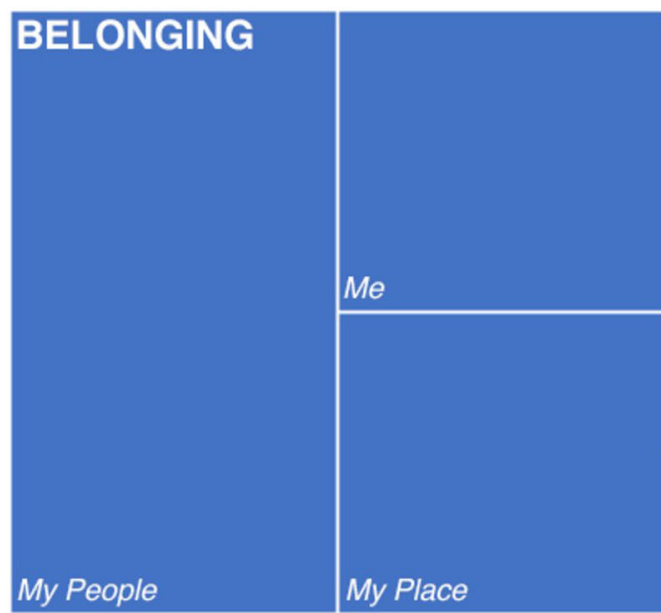

Fig. 6 Layer 1 coding density summary: Fellowship.

overview of how their micro-narratives were coded across these sub-categories:

For comparison purposes, an overview of the coding density of non-Fellowship comments is also summarised in Fig. 7.

The numbers of responses coded against different subcategories is summarised as follows, with non-Fellowship responses include for comparison (Table 1):

In general, the main themes to emerge from the thematic categorisation of narrative data were to do with social and personal benefits, spiritual/transcendental aspects, and enjoyment, corroborating the findings of the geometric analysis, which highlighted social impact alongside spiritual, mental and paramusical outcomes. This also partially validates the findings of a previous study (Skingley and Bungay 2010), which found similar themes of enjoyment and increased social interaction, as well as a similar de-emphasis of benefits to physical health, and a dissimilar emphasis on improved mental health and cognitive benefits. For the Fellowship participants, there is also a particular emphasis on a 'sense of place' in their narratives, and a de-emphasis on 'presentational performance', further corroborating the results of the geometric analysis.

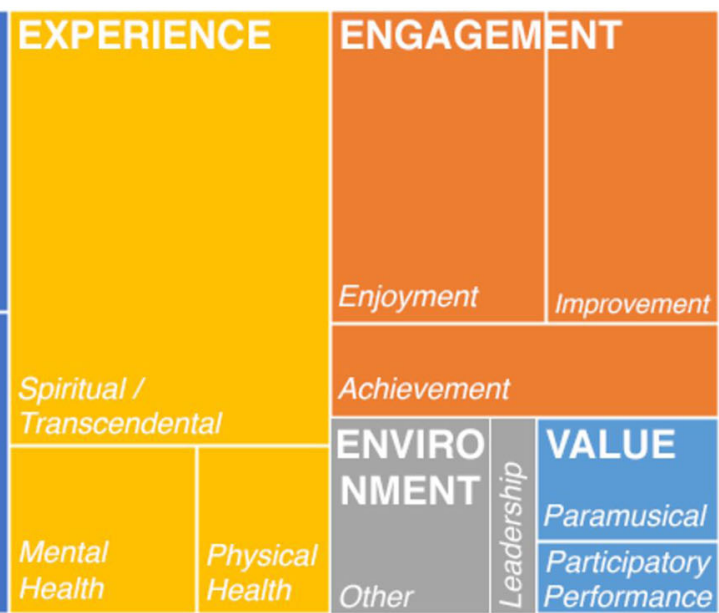

A range of participant responses are outlined in the following sections, which help to illuminate these themes, with particular attention paid to the various correspondences between the geometric and thematic analyses.

Belonging (Triad 1). Participant narratives emphasised the affordances of group singing to promote social cohesion and bonds of trust and attachment between people, with one Fellowship participant commenting 'The sense of camaraderie amongst the group became very strong through our experience today and I feel that I have made friends on a much deeper level'. (SM-75). ${ }^{8}$ This aligns with the narratives reflecting regular singing in community choirs as reported by non-fellowship participants, such as 'being part of a singing group is like being part of a special gang or clan. You enjoy seeing each other and spending time together. Each meeting brings you closer' (SM-27).

Experience (Triad 2). Fellowship participants communicated the strength of their experiences as something potent, which had a strongly positive spiritual and psychological dimension to it, 'an experience, which will stay with me forever'(SM-26). ${ }^{9}$ Another commented: 


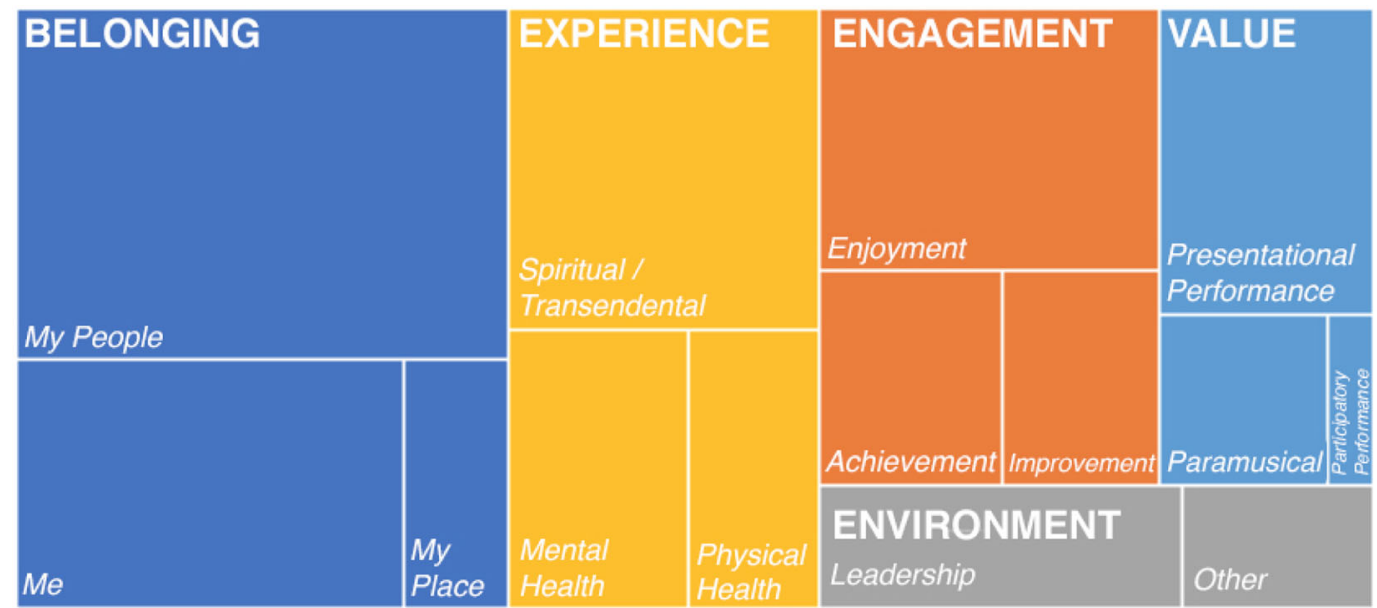

Fig. 7 Layer 1 coding density summary: non-Fellowship.

Table 1 Layer 1 thematic categorisation.

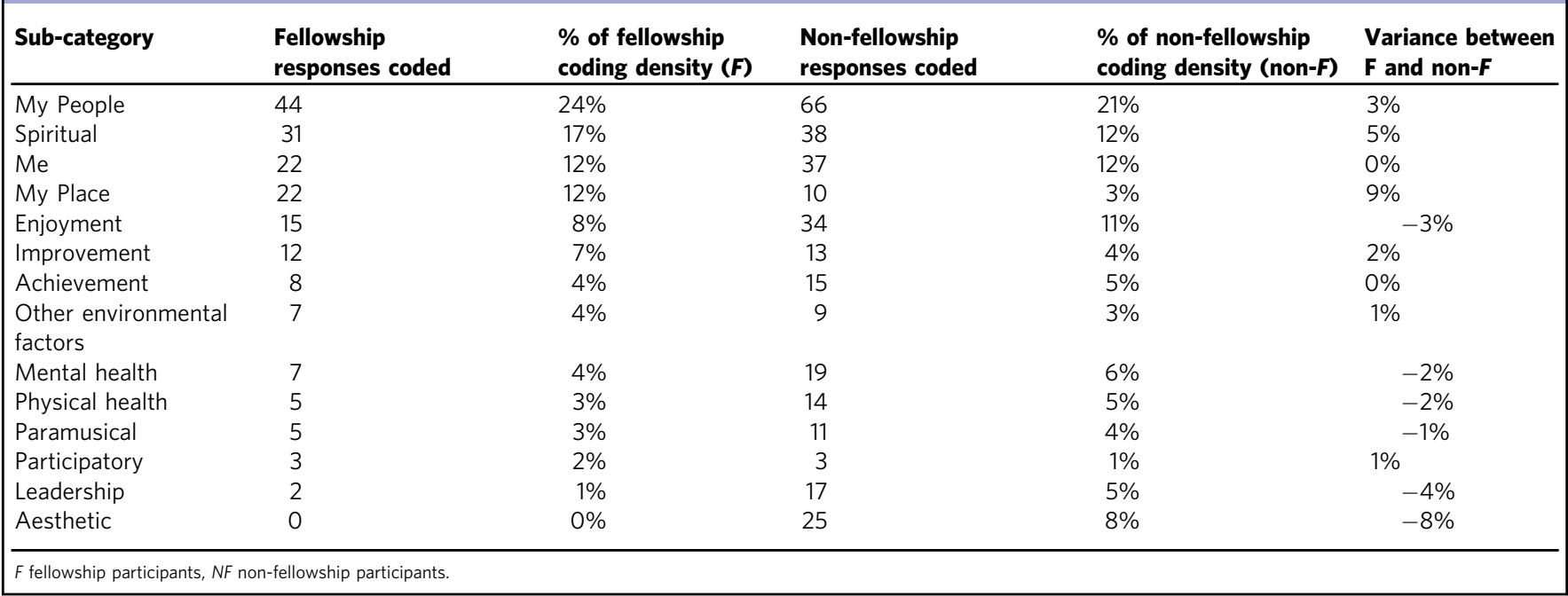

'together with your singing companions your voices soar and are heard-you become part of a magical beast that is 'Song'. You feel intoxicated almost, with the good feelings which are running through you. You want everyone to share in your happiness and to feel-if only for a moment - what you are feeling'. (SM-161)

Despite the physical strength and stamina required to participate in the Fellowship project, the stories of Fellowship participants also emphasised the spiritual and mental dimensions of the activity, and how singing together provided a means of structuring and organising their emotional responses to the activity: 'As I struggle to find meaning to my life following the death of my [spouse] the chance to sing with the Fellowship has given me purpose. For that I am forever grateful'. (SM-145) ${ }^{10}$

Value (Triad 4). Fellowship participants' stories emphasised both the aesthetic and participatory dimensions of their experiences: 'it doesn't matter if you're good at sight reading or learn by ear, loud or quiet, high or low-everyone has something to contribute and can all come together to create a meaning and beauty that is far, far greater than the sum of its parts'. (SM-38) ${ }^{11}$ Non-Fellowship participants emphasised similar issues, with one commenting, 'the surging harmonies that very large choirs can produce are sometimes almost too much to bear. It is at moments like this that I feel a visceral connection with every other singer in the hall'. (SM-61)

The stories of Fellowship participants highlighted the many 'paramusical' benefits of group singing, with one summarising their perception of the benefits: 'Increased confidence compassion and joy are obvious benefits to such events as this one but the most profound must be the sense of connection I alluded to earlier. To know our place in this world and smile at it'. (SM-94)

Discussion of analysis of distributed data generation/interpretation. From this initial geometric and thematic analysis, a number of key themes begin to emerge, pertinent to an understanding of how group singing might support the emergence and development of a 'healthy public'. While group singing may contribute to individual health and wellbeing, the primary benefit participants across both groups identified is the way that it brings them to a closer, more profound, connection with others. For the Fellowship participants in particular, while a sense of 'place', and a shared purpose certainly made an important contribution to the overall beneficial effects of singing together, the common thread in their narratives about group singing remained the sense of connection they felt with their fellow singers. One might therefore 
infer the experience of group singing as a form of 'communitas' (DeNora 2000, p. 149) or 'collective joy' (Turner 2012) invoking aspects of 'deep social mind' (Whiten 2007; Cozolino 2014) and mutual subjectification (Biesta 2017), driven by the power of social 'appearance' (Arendt 1977; Camlin 2018), i.e. 'showing up' for each other.

The phenomenon of 'entrainment' (Clayton 2012) may help to explain why music is particularly effective at building social bonds in this way. One possible interpretation of the 'beam' pattern of signification in the 'fitness landscape' of Triad 4: Value (Fig. 5) is that it illustrates a creative tension between the aesthetic and participatory musical dimensions of group singing, which appears to support the emergence of paramusical outcomes, i.e., that paramusical outcomes are enhanced when both aesthetic (performing 'works') and participatory (performing 'relationships') dimensions are engaged. Of particular interest is the difference between the significant emphasis placed on paramusical outcomes in the geometric analysis, when compared with a relative absence of discussion of such outcomes in participant narratives. Possible explanations for this might be to do with inconsistencies in coding, or with participants finding it harder to articulate these outcomes, a theme which is amplified in the results of the subsequent layer.

Thematic analysis of 'Theming' workshop. In the 'theming' workshop, a number of key themes were developed with participants through discussion of the data, emphasising a complex entanglement of contributory factors underpinning their experiences. At the end of the 3-h workshop, they co-created a list of themes reflecting their discussions, which generally validated the findings of the geometric analysis, and centred around themes of communitas; physical exertion; having fun and sounding good; a sense of place; transcendence through relationship.

Communitas. Participants talked about the sense of a 'shared goal' $\left(\mathrm{TW}-5^{12}\right.$ ) as well as 'strength and unity in adversity' (TW-7). The theme of connectivity and togetherness was emphasised: 'what comes through when you look at a lot of these [data], is that sense of connection between people' (TW-6). Another participant suggested that, 'the fellowship is more of a collective experience rather than an individual experience.' (TW-4) This led to discussions on some of the complex ways in which the social, musical and geographical dimensions of experience inter-relate. ${ }^{13}$

Physical exertion. There was general consensus that the reason the physical demands of the activity were de-emphasised in people's stories, and in the geometric data were to do with the physical exertion involved in the project being 'the price that's been paid forward for the experience' (TW-7). In other words, although the activity involved physical exertion, this was not experienced as distressing, because of the payoff of the other benefits arising.

Having fun and sounding good. The importance of fun and enjoyment was emphasised, and this sense of fun appeared to explain why respondents' awareness may have been less on the physical demands of the activity as well. This led into a protracted discussion about the aesthetic and participatory dimensions of music represented in Triad 4 , and why the paramusical benefits seemed to be important, as a means of uniting these dimensions: 'you want to make it sound good for everybody else, so that everyone's enjoying them[selves] off that experience. However, also because of the beauty around you as well' (TW-5). ${ }^{14}$ Striking a balance between these complementary musical dimensions of aesthetic qualities and fun activities seems to be important. As one participant expressed it, 'it's about making a good sound for people to listen to without losing the 'I'm singing for fun" (TW-4).

Sense of place. This complex entanglement of the aesthetic, the participatory and the paramusical also extended into the role the landscape itself played in the experience, and the connection participants felt 'to the mountains, to the hills and to nature' (TW-2). One participant commented, 'somehow it was the mountains that were reverberating with us, if you see what I mean, rather than an audience' (TW-2). This idea, of the location and the musical activity as complementary resources, that facilitated social connection in a complex way was echoed in comments about 'the way that the land is used to help people connect with each other' (TW-1), with another participant suggesting 'the National Trust is a conservation charity that looks after special places. However, not just because they're nice. As they're important for kind of what makes us human' (TW-6). This 'culturally enactive' (Cross and Woodruff 2009) dimension of the project's meaning-singing in fellowship as an act of commemoration of the sacrifice of the lives of previous generations who had also found fellowship in the land itself-was an important artistic feature of the project, and conditioned the meaning that participants made of their experiences.

Transcendence through relationship. The importance of the idea of 'relationship' implicit within the act of group singing began to crystallise within this discussion, although it was not easy for participants to articulate. As the discussion evolved, the complexity of the subject began to slowly emerge, especially in relation to the 'strongest', most transcendental experiences. One of the workshop participants reflected, 'we talked a lot about the feeling, about how sometimes-well, certainly for me, the stronger the feeling and the sensation was about that connection, the less able I was to articulate it in the stories' (TW-10), and one participant asking, 'so what is it that we haven't got the language for, but feel?' (TW-5)

Discussion of thematic analysis of 'Theming' workshop. Taking this theme of elusiveness further, if one accepts the idea of music as a 'polyvalent' system, perhaps the more these different valences are activated, the harder it becomes to articulate. The language of the participants point to this elusive complexity; at one point one of them describes it as, 'that thing that everybody knows but nobody knows' (TW-4), and elsewhere in the discussion another talks about, 'this unnameable something that we name by what it is not rather than what it is.' (TW-5).

Rather than rejecting this kind of talk about music's slippery meaning as being too nebulous to be useful to our discussions, one should perhaps recognise that the 'simultaneous multiplicity of meaning' (Bowman 2004, p. 30), which underpins the participants' intuitive responses is perhaps one of music's defining characteristics, and needs embracing:

'to the extent it refuses to reduce plurality and diversity to attributes of some unified entity, polysemy is inherently ambiguous: and yet, polysemic constellations of meaning are no less vivid, rich, or potent for being multifaceted' (Bowman 2004, p. 30)

Indeed, the way that participants talk about their experience perhaps points to 'pre-linguistic' mechanisms, which go beyond the capacity of language to fully articulate, where the music itself is a form of communication that language cannot be a substitute for. Pavlicevic (2013b) refers to these kinds of transcendental experiences as 'magic moments' where 'the group is in peak flow,' and the 'social-musical improvisation [seems] to be known within and between all minds and bodies as one, complex, phenomenon' 
(p. 102). Elsewhere she suggests these moments occur when identities are dissolved (or shared) in the interests of being people together in music in this place and in this time' (Pavlicevic 2013a, p. 196). These descriptions of group singing as a transcendental, spiritual experience are not uncommon in participants' narratives about their experience (Clift and Hancox 2001; Dingle et al. 2013). It is perhaps these moments of 'peak flow', which contain the most significance for participants, where their experience of 'self-other merging' (Tarr et al. 2014) and interpersonal 'resonance' (Lewis et al. 2001; Siegel 2011) are strongest. As one respondent put it, 'singing with others takes me out of myself into another space. I felt my precious sense of self drifting away on a wave of harmony' (SM-91).

Thematic analysis of unstructured interview. The purpose of this unstructured interview with two participants from professional therapeutic backgrounds was to consider some of these more 'elusive' sentiments, which had arisen in the Layer 2 focus group discussion in more detail. During the interview, the participants were invited to reflect on their first hand experience of interpersonal 'attunement' $-\mathrm{a}$ recognised theme within psychotherapeutic contexts-in both professional therapeutic and group singing contexts.

The 'therapeutic alliance' between therapist and client-where the therapist 'voluntarily [enters] into a kinship relationship with the patient' (Clarkson 1992, p. 294) lies at the heart of therapeutic work. For the therapist, this includes managing the powerful phenomena of both transference-'the process by which a patient displaces onto [his] analyst feelings, ideas, etc., which derive from previous figures in [his] life' (Rycroft 1995, p. 168)-and countertransference, 'the analyst's transference on [his] patient' (p. 25). The power of the therapeutic alliance lies in navigating these relational complexities: 'resonance requires that we remain differentiated - that we know who we are-while also becoming linked' (Siegel 2011).

Transference. For both of the therapists interviewed-sensitised to such matters of energetic 'transfer'-group singing provides a way to experience others without being drawn into a more overtly 'therapeutic' mindset or psychologically intimate encounter. Therapist 2 (T2) suggested that the singing group was somewhere where, 'we don't get hit by those waves of transference that can pull you all over the place and make you feel clearly off.' Asked explicitly to reflect on any similarities and differences in experience of relationships in both singing and their therapy work, T2 reflected, 'I think it's probably the same process. However, the amount that I open up [and] utilise that process [in group singing] is controlled by me.' Group singing therefore offers a different kind of relationship to that experienced in everyday life, as T1 elaborated:

'I suspect that the deep interaction which occurs when groups sing together has always provided a safe way to bond emotionally and socially with our community, offering respite from the more complex interactions we negotiate in our close relationships and work life.'

Safe danger. This led into a discussion of how the activity of group singing might provide a 'protective frame' (Apter 2007, pp. $50-53$ ) around the experience of relationship, which one of the interviewees characterised as 'safe danger':

'I think that when we're singing together, we are in a safe state. You can be with other people, and [they're] not a threat. There is something very intense about some of the experience. But it's not stressful. It's not unsafe. It feels very safe' (T1). ${ }^{15}$

The idea of 'safe danger' may sound similar to Higgins' idea of 'safety-without-safety', a feature of improvisational music workshop practice where, 'boundaries are marked to provide enough structural energy for the session to begin, but care is then taken to ensure that not too many restraints are employed that might delimit the flow or the becoming of any music-making' (Higgins 2008 , p. 331). However, in these group singing activities, it is a psychological safety-rather than, or as well as musical safetywhich is emphasised, where people are afforded the opportunity to experience the healing potential of feeling connected with others (von Lob et al. 2010, p. 49), but without the deep intensity of intimacy becoming overwhelming.

While the small sample size $(n=2)$ of the therapist interview makes it too small to be generalisable, the idea that group singing provides an opportunity for participants to rehearse and perform 'healthy relationships' under the conditions of 'safe danger' is worth testing in future research, and could potentially enhance existing knowledge about group singing and emotion regulation (Dingle et al. 2017).

\section{Discussion}

This study reveals the sheer complexity of what may be activated during group singing. It works at both an individual and a group level, alongside culturally situated factors: the performance of specific works or behaviours, a common purpose and a sense of place or occasion, can all contribute to what the resulting music 'means', which in turn contributes to the overall beneficial effects of singing together.

Essential characteristics of group singing. A number of overarching themes emerge from the study in relation to the more invariant characteristics of group singing; the significance of 'communitas' and moments of transcendence, as well as an extended notion of performance, which includes the performance of 'healthy' relationships.

The idea of 'communitas' is perhaps a stronger expression of the social impact of group singing than that of building social capital, or social bonding/cohesion, as it carries with it the sense of 'collective joy' (Turner 2012), which appears to lie at the heart of the activity. Losing oneself in these collective moments of transcendence also seems to be an essential part of the experience, the 'self-other merging' (Tarr et al. 2014) involved creating the conditions of 'safe danger' wherein participants can rehearse and perform a different experience of relationship. From the sense that participants make of their experiences, we suggest that it is an adaptive complex of interdependent musical, neurobiological and psychosocial mechanisms, which underpins such transcendence. While testing this hypothesis was beyond the scope of the study, we hope we have highlighted the need for interdisciplinary approaches, which include the descriptive and reflective selfawareness of the participants themselves, to enable these complex systems to be understood.

At the heart of group singing is therefore the idea of performance, but it is perhaps a more nuanced notion of performance than is commonly discussed, where it is 'more about the social relations being realised through the performance than about producing art that can somehow be abstracted from those social relations,' (Turino 2008, p. 36). A musical performance is usually about performing recognisable works, but it is also about performing relationships-either in service of the realisation of those works, or as a purely relational activity performed for each other with no audience present (Camlin 2020, 2018). Participants' 
responses to the question of significance of the aesthetic and participatory dimensions of music represented in Triad 4 (Value) provide a strong visual correlate of this idea that music's power lies in harnessing both of these dimensions. This creative tension may be instrumental in the conditions for 'peak flow' in music, and help to explain how music's paramusical benefits come about. Participants' stories describe a fully aesthetic experience, i.e., where their senses are 'operating at their peak' (Robinson 2010), but the focus is perhaps more on the activity itself rather than on an audience's perception of it (Turino 2008, p. 28). Greater immersion in the fun and relational aspects of group singing might mean that people worry less about the sound they make, which may in turn support them to sing from a more relaxed place, and paradoxically may therefore contribute to a more aesthetically satisfying performance.

Performing healthy relationships. Fundamentally, healthy relationships underpin healthy publics. Group singing provides participants with an opportunity to encounter others in meaningful ways, which foster connection, resonance and attunement, and is a way of building personal and collective resources for health and wellbeing. Group singing may provide a valuable way of structuring opportunities for 'limbic resonance' (Lewis et al. 2001, pp. 169-170), and for enhancing the effects of such resonance, because musical entrainment both benefits from, and contributes to, a synchronisation of physiology_breathing and movement, as well as rhythmic and harmonic synchronisationwhich are in turn symptomatic of interpersonal neurobiological attunement (Siegel 2016, p. 61).

Because of the very present sense in which people need to attune to one another to perform well, one might say that group singing supports participants to experience and 'perform' healthy relationships within a protective frame of 'safe danger' where they can explore the boundaries of social connection in nonthreatening ways, and literally 'hear' when the relationship is performing well, by the sounds which are co-produced. From the point of view of the participants, the way in which they come to feel a resonant connection with other individuals, with the group as a whole, with the music they are singing and its meaning, and their surroundings, appears to lie at the heart of what they feel is most powerful about the group singing experience. The wellbeing effect may also be enhanced because participants are aware of it when it happens; when these powerful feelings, which transcend the 'magical' musical moment are 'felt' (Siegel D (2015) 'Interview with Dan Siegel (personal communication) and shared with other participants, and with audiences.

Significantly, this experience of an 'idealised' form of relationship is one which participants appear able to mobilise as a resource for use in their everyday lives, as suggested by Dingle et al. (2013). As one participant put it, 'you can gain confidence which supports many other areas of your life. You get to know others in the community and you feel actively part of the place where you live' (SM-60). Group singing might therefore afford considerable potential for 'mutual recovery', i.e., 'a mainstream mechanism for social connectedness and recovery for all involved in healthcare, that is, the healthcare workforce alongside patients and informal carers' (Crawford et al. 2015, p. 137), because it can represent a very accessible and efficient way to co-create psychological resources that people can take with them out of the rehearsal room or the performance venue and into their everyday lives. ${ }^{16}$

Reflection on methodology. This study shows that Sensemaker ${ }^{\circledast}$ (Snowden 2016) provides a valuable 'qualiquant' method for building understanding that is grounded in participant experience yet able to identify trends and patterns of experience beyond that of the individual, effectively addressing the call to look at 'the actual experience of culture and the arts rather than the ancillary effects of this experience' (Crossick and Kaszynska 2016, p. 21). While a more scientific approach to understanding the power of group singing-through the isolation and understanding of individual phenomena-plays an important part in understanding its value, on its own such an approach may be insufficient, because to isolate any particular effect is potentially to miss the totality of the experience and the interconnectedness of the cultural, social and musical dimensions, which ground its significance. Both scientific and experiential perspectives on group singing are therefore essential to gain an understanding of its value, and neither is sufficient on its own. A deeper exploration of this sympathetic entanglement of group singing's 'polyvalent significance' therefore requires an interdisciplinary approach, mobilising insights from fields as diverse as Neuroscience, Ethnography, Entrainment Studies and Health Humanities.

Limitations. There are a number of limitations to the study. The overall population $(n=78)$ of the study is not large, even when combined with the comparison group $(n=88)$, and representative of only a small number of those who regularly sing in a group. Any inferential judgements about the value of group singing more generally to a broader population are therefore speculative. The study was conducted as a form of practice-asresearch (Nelson 2013) with most of the participants $(n=137)$ participating in singing activity led by one of the members of vocal group Mouthful, and one of the researchers observing, participating in and leading some of the group singing activity, with all of the attendant complications of subjective influence which that entails. Coding was only undertaken by one researcher, again potentially introducing further unconscious bias, although this was mitigated by the inclusion of a 'theming' workshop where participants were able to contribute to the process of thematic analysis at the same time as enhancing their descriptive self-awareness.

There are also some underpinning philosophical and sociological limitations, which warrant further investigation. Singing appears to be good in a number of ways for those who participate in it, but what about those who feel unable to participate in it, or who simply don't want to? Is singing only good for those who it's good for? What are the strongest drivers of wellbeing arising from participation, and how does group singing reinforce or interfere with these in people with different personalities, experiences or backgrounds? What factors limit the experience of the effect, and under what circumstances might group singing even be a source of negative experience, rather than the positive one reported by participants in this study? And is the experience the same with different music? Or with different practitioner styles? These questions would be important to explore in a further study, and indeed a more experiential approach would enable rigorous exploration of the variables of practice approach and repertoire.

The idea of 'entanglement' between musical and neurobiological entrainment throws up some particular challenges. If the activity of group singing provides a space for people to rehearse and perform 'healthy attachment' (Bowlby 1988) to others-in both musical and social terms-is such attachment an outcome of the activity, a pre-requisite for it, or both? As an activity, is it more accessible to those with more experience of healthy attachment in the first place, or those willing to seek it out? For those experiencing social anxiety, the idea of revealing yourself intimately through your voice to a large group of strangers might be terrifying, in the same way that the same activity appears to be an almost spiritual one for those with the confidence and 
capability to participate in it. Could it be that one of the reasons that group singing appears to exclude some people from it is precisely because it is entangled with interpersonal neurobiological intimacy? If one views group singing as a celebration of the ability of those who participate in it to demonstrate their capacity for healthy attachment, then where such attachment has been impaired, the intimacy of musical entrainment may be uncomfortable or even distressing. Under what circumstances might group singing be therefore viewed as an act of 'symbolic violence' (Bourdieu 1992; Schubert 2012) against those lacking the experience of healthy attachment or social confidence to involve themselves in it? And how can group singing activities be structured to ensure that those most in need of its benefits are able to participate in it? While there is evidence of the positive impact of group singing on mental health recovery (Clift and Morrison 2011; Coulton et al. 2015; Shakespeare and Whieldon 2017; Williams et al. 2018), the focus is often on those possessing the willingness and capacity to participate in the first instance, rather than those who may choose not to because they believe they would find it too exposing, and/or raise too much anxiety for them. A future focus on the motivations of those who choose not to participate in group singing would help to understand these issues more clearly.

To fully test the ideas emerging from the study about music's polyvalent complexity was beyond the scope of the study, and the process of doing so may have limited the findings to some extent. Experiments could be designed to triangulate the qualitative data with more measurable outcomes such as rhythmic/harmonic analysis in the case of musical entrainment, or HRV/MNS/ brainwave synchronisation in the case of interpersonal neurobiological attunement, but would require a much larger study involving researchers from other disciplines. We discuss some of the methodological complexities of devising protocols to address the entangled complexities of such a study elsewhere (Daffern et al. 2018).

\section{Conclusions}

This study enhances existing knowledge of the benefits of group singing (Williams et al. 2018; Dingle et al. 2019), and the psychosocial and psychobiological mechanisms which underpin it (von Lob et al. 2010; Fancourt et al. 2016), by pioneering the use of Sensemaker ${ }^{\circledast}$ as a software tool to investigate group singing as a 'complex adaptive system' from the point of view of individual experience. The study highlights the relative stability of the 'social bonding' effect of group singing-an apparently invariant characteristic of group singing-even in the face of other potentially disrupting influences such as the impact of outdoor exercise on the experience. It may be that singing is good for the physical health of the singers in the study, but from their accounts, the reason they sing together is because it provides opportunities for deep levels of connection - to others, to their environment, and to themselves. As one respondent expressed it, 'singing together has enabled us to meet each other as human beings differently' (SM-102).

The use of Sensemaker ${ }^{\circledR}$ as a method enables this phenomenon to be more easily identified and distinguished from other effects. The study highlights the potential of Sensemaker ${ }^{\circledR}$ or similar methods as a valuable means to collect qualitative data at a large scale, potentially leading to more generalisable findings. Developing this methodology therefore has the potential to make a valuable contribution to a deeper understanding of cultural value.

The emerging hypothesis that the health benefits associated with group singing are underscored by a complex interdependence between musical, neurobiological and psychosocial mechanisms warrants further investigation. The study also highlights an opportunity for future research design to 'frontload' participant experience in order to explore these complexities. A larger study could test the findings more comprehensively, especially when combined with measures from other disciplines to verify or triangulate results.

This work presents a strong case for group singing as healthy public, contextualising a new qualitative approach within existing evidence from empirical investigations and practice-led research. If practitioners and facilitators of group singing-as well as researchers and commissioners-approach it with an awareness that its potential as a health resource are to be found within an adaptive complex of effects and experiences, a better understanding of these complex interdependencies will emerge over time.

\section{Data availability}

The data sets generated during and/or analysed during the current study are not publicly available due to ethical considerations to comply with EU General Data Protection Regulations (GDPR), but are available from the corresponding author on reasonable request.

Received: 24 September 2019; Accepted: 13 July 2020;

Published online: 05 August 2020

\section{Notes}

1 'to take part, in any capacity, in a musical performance, whether by performing, by listening, by rehearsing or practicing, by providing material for performance (what is called composing), or by dancing.' (Small 1998, p. 9)

2 Moreover, bearing some similarity to the thematic categorisation of participant experience into personal impact, social impact and functional outcomes (Dingle et al. 2013, p. 8).

3 An award-winning project commissioned by the UK National Trust and funded by AHRC and Arts Council England, which involved the vocal group Mouthful taking large groups of singers onto UK mountains summits to perform a song cycle commemorating the gift of land containing 14 mountain summits by a local mountaineering group in 1923.

4 including one of the researchers.

5 Signifier sets 1 (Belonging), 2 (Experience), and 6 (Transformation) were 'polymorphic'.

6 Participants were able to choose not to record a mark for any, or all, of the signifier sets by selecting $\mathrm{n} / \mathrm{a}$ '.

7 Sensemaker ${ }^{\oplus}$ participant identifier.

8 Further examples of these stories can be found at https://docs.google.com/document/ $\mathrm{d} / 1$ 1o1NK9rfOSFWOCthsicTgOFml2GjcPWZXayiZ3JubHUE/edit\#heading $=\mathrm{h}$ tj5bk549m9by.

9 Further examples can be found at https://docs.google.com/document/d/ 1o1NK9rfOSFWOCthsicTgOFml2GjcPWZXayiZ3JubHUE/edit\#bookmark=id. lnd8dc70zqx1.

10 Further examples can be found at https://docs.google.com/document/d/ 1o1NK9rfOSFWOCthsicTgOFml2GjcPWZXayiZ3JubHUE/edit\#heading $=\mathrm{h}$ r0wueos210d.

11 Further examples can be found at https://docs.google.com/document/d/ 1o1NK9rfOSFWOCthsicTgOFml2GjcPWZXayiZ3JubHUE/edit\#bookmarkid. br5m3iip $4 x c 8$.

12 Focus Group Theming Workshop participant no.

13 More of the discussion can be found at https://docs.google.com/document/d/ 1o1NK9rfOSFWOCthsicTgOFml2GjcPWZXayiZ3JubHUE/ edit\#heading=h.6yig576iyjg5.

14 A fuller account of this discussion is available at https://docs.google.com/document/ d/1o1NK9rfOSFWOCthsicTgOFml2GjcPWZXayiZ3JubHUE/edit\#heading=h. xhlxlyfd32cx.

15 A longer discussion of this issue can be found at https://docs.google.com/document/ d/1olNK9rfOSFWOCthsicTgOFml2GjcPWZXayiZ3JubHUE/edit\#heading=h. rk2liff5z2cs.

16 A comment from one of the two therapist interviewees illustrates this: https://docs. google.com/document/d/1o1NK9rfOSFWOCthsicTgOFml2GjcPWZXayiZ3JubHUE/ edit\#heading=\%20h.rhl6p0kcqtmz. 


\section{References}

Apter MJ (2007) Reversal theory: the dynamics of motivation, emotion and personality, New edition. Oneworld Publications, Oxford

Arendt H (1977) The human condition. University of Chicago Press, Chicago, Translated by M. Canovan

Bailey B, Davidson J (2005) Effects of group singing and performance for marginalized and middleclass singers. Psychol Music 33(3):269-303. https://doi. org/10.1177/0305735605053734

Barenboim D (2009) Everything is connected: the power of music. Phoenix, London

Bartleet B-L (2016) The role of love in intercultural arts theory and practice. In: Burnard P, Mackinlay E, Powell K (eds) The Routledge International Handbook of Intercultural Arts Practice. Routledge, New York, pp. 91-101

Bartleet B-L, Higgins L (2018) An overview of community music in the twenty-first century. In: Bartleet B-L, Higgins L (eds) Oxford Handbook of Community Music. Oxford University Press, New York

BBC Health Check (2018) Singing for health, BBC. Available at https://www.bbc. co.uk/programmes/w3cswjkk (Accessed 21 Jun 2018)

Belfiore E, Bennett O (2008) The social impact of the arts: an intellectual history. Palgrave Macmillan, London

Biesta G (2017) The rediscovery of teaching, 1 edition. Routledge, New York

Bithell C (2014) A different voice, a different song: reclaiming community through the natural voice and world song. OUP USA, Oxford; New York

Bourdieu P (1992) Language and symbolic power. Polity Press, Cambridge

Bowlby J (1988) A secure base. Routledge, London; New York

Bowman, Wayne. 2004. 'Cognition and the Body: Perspectives from Music Education'. In: Bresler, L (ed.) Knowing Bodies, Moving Minds: Towards Embodied Teaching and Learning. Dordrecht, Netherlands: Kluwer Academic Publishers

Brown $\mathrm{R}$ et al. (2017) Ternary graph as a questionnaire: a new approach to assessment of quality of life. Brit J Oral Max Surg 55(7):679-684. https://doi. org/10.1016/j.bjoms.2017.04.011

Buglass G, Webster M (2004) Finding voices, making choices: creativity for social change. Educational Heretics Press, Shrewsbury

Burkeman O (2015) 'Want to be happy? Join a choir', The Guardian, 18 December. Available at http://www.theguardian.com/lifeandstyle/2015/dec/18/whysinging-makes-people-happy-oliver-burkeman?CMP $=$ share_btn_fb (Accessed 20 Dec 2015)

Camlin DA (2020) Organisational dynamics in community ensembles. In: Daffern, H, Bailes, F, Timmers, R (eds) Together in music. Oxford University Press, Sheffield

Camlin DA (2014) Whose quality is it anyway? J Arts Commun 6(2+3):99-118 https://doi.org/10.1386/jaac.6.2-3.99_1

Camlin DA (2016) Music In Three Dimensions. Doctoral Thesis. University of Sunderland

Camlin DA (2018) 'Assessing quality in socially engaged musical performances'. In: Reflective conservatoire. Reflective Conservatoire. Barbican-Guildhall, London

Camlin DA (2019) 'Recovering our humanity: what's love (and music) got to do with it?' In: International Fjord Summer School 2019, International Fjord Summer School, Grieg Research School in Interdisciplinary Music Studies, Bergen. Available at https:/www.uib.no/en/rs/grieg/122694/exploringartistic-pedagogic-and-therapeutic-practices-interdisciplinary-knowledges

Chernoff JM (1979) African rhythm and African sensibility: aesthetics and social action In African musical idioms. University of Chicago Press, Chicago, IL

Clarkson P (1992) Transactional analysis psychotherapy: an integrated approach. Routledge, New York

Clayton M (2012) What is entrainment? definition and applications in musical research. Empir Musicol Rev 7(1-2):49-56. https://doi.org/10.18061/1811/52979

Clayton M, Will U, Sager R (2004) In time with the music-the concept of entrainment and its significance for ethnomusicology. ESEM CounterPoint $1: 1-84$

Clift S et al. (2013) An evaluation of community singing for people with COPD (chronic obstructive pulmonary disease). Canterbury Christ Church University, Canterbury, UK

Clift S (2013) 'Singing, wellbeing and health'. In: Music, health and wellbeing. Oxford University Press, Oxford, UK

Clift SM, Hancox G (2001) The perceived benefits of singing findings from preliminary surveys of a university college choral society. J R Soc Promot Health 121(4):248-256. https://doi.org/10.1177/146642400112100409

Clift S, Manship S, Stephens L (2017) Further evidence that singing fosters mental health and wellbeing: findings from the West Kent and Medway project. Soc Incl Ment Health 21(1):53-62

Clift S, Morrison I (2011) Group singing fosters mental health and wellbeing: findings from the East Kent "singing for health" network project. Men Health Soc Incl 15(2):88-97. https://doi.org/10.1108/20428301111140930

Cognitive Edge (2018) The Making of Meaning. Available at https:// emergentmeaning.wordpress.com/ (Accessed 29 Jun 2018)
Coulton S et al. (2015) Effectiveness and cost-effectiveness of community singing on mental health-related quality of life of older people: randomised controlled trial. Br J Psychiatr 207(3):250-255. https://doi.org/10.1192/bjp.bp.113.129908

Cozolino L (2014) The neuroscience of human relationships: attachment and the developing social brain. W. W. Norton \& Company, New York, USA

Crawford P et al. (2013) Creative practice as mutual recovery in mental health, Ment Health Rev J, 18. https://doi.org/10.1108/MHRJ-11-2012-0031

Crawford P et al. (2015) Health humanities, 2015 edition. Palgrave Macmillan, London; New York

Creech A (2014) Active ageing with music: supporting wellbeing in the third and fourth ages. Institute of Education Press, Dulles, TX

Cross I, Woodruff GE (2009) Music as a communicative medium. In: Botha R, Knight C (eds) The prehistory of language. Oxford University Press, Oxford, pp. 113-144

Crossick G, Kaszynska P (2016) Understanding the value of arts and culture: the AHRC cultural value report. Arts \& Humanities Research Council, Swindon, UK, http:// www.ahrc.ac.uk/documents/publications/cultural-value-project-final-report/

Daffern H (2017) Blend in singing ensemble performance: vibrato production in a vocal quartet. J Voice 31(3):385.e23-385.e29. https://doi.org/10.1016/j.jvoice.2016.09.007

Daffern $\mathrm{H}$ et al. (2018) Exploring the potential of virtual reality technology to investigate the health and well being benefits of group singing. Int J Performance Art Digit Media 15(1):1-22. https://doi.org/10.1080/14794713.2018.1558807

DeNora T (2000) Music in everyday life. Cambridge University Press, Cambridge

DeNora T (2013) Music asylums: wellbeing through music in everyday life. Ashgate, Farnham, UK

Dingle, GA et al. (2013) "To be heard": the social and mental health benefits of choir singing for disadvantaged adults, Psychol Music, 41. https://doi.org/ $10.1177 / 0305735611430081$

Dingle $G$ et al. (2017) Choir singing and creative writing enhance emotion regulation in adults with chronic mental health conditions. Br J Clin Psychol 56 (4):443-457. https://doi.org/10.1111/bjc.12149

Dingle G et al. (2019) An agenda for best practice research on group singing, health, and well-being. Music Sci 2:1-15. https://doi.org/10.1177/2059204319861719

Dunbar PR (2013) The science of love and betrayal. Faber \& Faber, London

Elliott DJ (1995) Music matters: a new philosophy of music education. OUP USA, New York/Oxford

Elliott DJ, Silverman M (2013) Why music matters: philosophical and cultura foundations. In: MacDonald, R, Kreutz, G and Mitchell, L (eds) Music, health and wellbeing. Oxford: Oxford University Press, pp. 942-1447

Elliott DJ, Silverman M (2014) Music matters: a philosophy of music education. Oxford University Press, New York, USA

Elliott D, Silverman M, Bowman W (2016) Artistic citizenship: artistry, socia responsibility, and ethical praxis. Oxford University Press, New York, USA

Eno B (2008) Singing: the key to a long life, NPR.org. Available at http://www.npr org/templates/story/story.php?storyId=97320958 (Accessed 10 Apr 2014)

Fancourt D et al. (2016) Singing modulates mood, stress, cortisol, cytokine and neuropeptide activity in cancer patients and carers, ecancer, 10(631). https:// doi.org/10.3332/ecancer.2016.631

Gabrielsson A (2011) Strong experiences with music: music is much more than just music. Oxford University Press, Oxford; New York

Hallam S (2015) The power of music: a research synthesis on the impact of actively making music on the intellectual, social and personal development of children and young people. International Music Education Research Centre (iMERC), London

Higgins L (2008) The creative music workshop: event, facilitation, gift. Int J Music Educ 26(4):326-338. https://doi.org/10.1177/0255761408096074

Hinchliffe S et al. (2018) Healthy publics: enabling cultures and environments for health. Palgrave Commun 4(1):57. https://doi.org/10.1057/s41599-018-0113-9

Holt-Lunstad J et al. (2015) Loneliness and social isolation as risk factors for mortality: a meta-analytic review. Perspect Psychol Sci 10(2):227-237

Keil C (1987) Participatory discrepencies and the power of music. Cult Anthropol 2 (3):275-283. https://doi.org/10.1525/can.1987.2.3.02a00010

Kreutz G et al. (2003) 'Does singing provide health benefits?'. In: 5th Triennial ESCOM Conference, Hanover University

Kreutz G et al. (2004) Effects of choir singing or listening on secretory immunoglobulin A, cortisol, and emotional state. J Behav Med 27(6):623-635 https://doi.org/10.1007/s10865-004-0006-9

Levitin D, Mallik A, Chanda, ML (2017) 'Anhedonia to music and mu-opioids: evidence from the administration of naltrexone', www.nature.com, 7(41952). https://doi.org/10.1038/srep41952

Lewis T, Amini F, Lannon R (2001) A general theory of love. Vintage Books, New York

Livesey L et al. (2012) Benefits of choral singing for social and mental wellbeing: qualitative findings from a cross-national survey of choir members. J Public Ment Health 11(1):10-26. https://doi.org/10.1108/1746572121207275

von Lob G, Camic P, Clift S (2010) The use of singing in a group as a response to adverse life events. Int J Mental Health Promot 12(13):45-53. https://doi.org/ $10.1080 / 14623730.2010 .9721818$ 
Macdonald K, Macdonald T-M (2010) The peptide that binds: a systematic review of oxytocin and its prosocial effects in humans. Harvard Rev Psychiatr 18 (1):1-21. https://doi.org/10.3109/10673220903523615

Malloch S, Trevarthen C (2010) Communicative musicality: exploring the basis of human companionship. Oxford University Press, Oxford

Miell D, MacDonald R, Hargreaves DJ (eds) (2005) Music Commun. Oxford University Press, Oxford

Mithen S (2007) The singing Neanderthals: the origins of music, language, mind, and body. Harvard University Press, Cambridge, MA

Molnar-Szakacs I, Overy K (2006) Music and mirror neurons: from motion to'e'motion. Soc Cognit Affect Neurosci 1(3):235-241. https://doi.org/ $10.1093 / \mathrm{scan} / \mathrm{nsl} 029$

Morrison I, Clift S (2013) A UK feasibility study on the value of singing for people with Chronic Obstructive Pulmonary Disease (COPD). UNESCO Observat Multi-Discipl J Arts 3(3):1-19

Mouthful (2020) The Mouthful Way, The Mouthful Way. Available at https:// www.mouthfulway.co.uk/ (Accessed 27 Jul 2020)

National Trust (2018) Songs on the Summits 1918-2018, National Trust. Available at https://www.nationaltrust.org.uk/borrowdale-and-derwent-water/features/ songs-on-the-summits-1918-2018 (Accessed 14 Mar 2018)

Natural Voice Network (2020) Natural Voice Network. Available at http://www. naturalvoice.net/ (Accessed 27 Jul 2020)

Nelson R (2013) Practice as research in the arts: principles, protocols, pedagogies, resistances. Palgrave Macmillan, New York

NICE (2015) Older people-independence and mental wellbeing. Guidelines NG32. National Institute for Health and Care Excellence, London. Available at http://www.nice.org.uk/guidance/ng32/resources/older-peopleindependence-and-mental-wellbeing-1837389003973 (Accessed 24 Jan 2016)

Pavlicevic M (2013a) Between beats: group music therapy transforming people and places. In: MacDonald R, Kreutz G, Mitchell L (eds) Music health and wellbeing. Oxford University Press, Oxford, pp. 196-212

Pavlicevic M (2013b) Let the music work: optimal moments of collaborative musicing. In: Stige B et al. (eds), Where Music Helps. Ashgate, pp. 100-123

Plowright D (2010) Using mixed methods: frameworks for an integrated methodology. Sage Publications Ltd, Los Angeles, London, New Delhi, Singapore, Washington DC

Robinson K (2010) Changing Education Paradigms, RSA Animate. Available at https://www.youtube.com/watch?v=zDZFcDGpL4U

Ryan RM, Deci EL (2001) On happiness and human potentials: a review of research on hedonic and eudaimonic well-being. Ann Rev Psychol 52:141-166

Rycroft C (1995) A critical dictionary of psychoanalysis. Penguin, London, UK; New York, USA

Särkämö T et al. (2013) Cognitive, emotional, and social benefits of regular musical activities in early dementia: randomized controlled study. J Gerontol 54 (4):634-650. https://doi.org/10.1093/geront/gnt100

Schubert JD (2012) Suffering/symbolic violence. In: Grenfell M (ed) Pierre Bourdieu: key concepts. Routledge, Durham

Shakespeare T, Whieldon, A (2017) Sing Your Heart Out: community singing as part of mental health recovery. Norfolk: University of East Anglia. Available at https://ueaeprints.uea.ac.uk/65446/1/Accepted_manuscript.pdf

Siegel D (2011) Mindsight: transform your brain with the new science of kindness. Oneworld Publications, Oxford

Siegel DJ (2012) Pocket guide to interpersonal neurobiology: an integrative handbook of the mind. W. W. Norton \& Company, New York, USA; London, UK

Siegel DJ (2016) Mind: a journey to the heart of being human. W. W. Norton \& Company, New York

Skingley A et al. (2011) The effectiveness and cost-effectiveness of a participative community singing programme as a health promotion initiative for older people: protocol for a randomised controlled trial. BMC Public Health 11 (142):1-22. https://doi.org/10.1186/1471-2458-11-142

Skingley A, Bungay H (2010) The Silver Song Club Project: singing to promote the health of older people. Br J Commun Nurs 15(3):135-140. https://doi.org/ 10.12968/bjen.2010.15.3.46902

Skingley A, Bungay H, Clift S (2011) Researching participatory arts, well-being and health: some methodological issues. J Arts Commun 3(1):73-87. https://doi. org/10.1386/jaac.3.1.73_1

Sloboda JA (1986) The musical mind: the cognitive psychology of music. Oxford University Press, Oxford

Small C (1998) Musicking: the meanings of performing and listening. Wesleyan University Press, Middletown, CT

Snowden D (2011) Naturalizing sensemaking. In: Mosier K, Fischer U (eds) Informed by knowledge: expert performance in complex situations. Psychology Press, New York; London, p.p 233-234

Snowden D (2016) Ethnography part II, cognitive edge. Available at http:// cognitive-edge.com/blog/ethnography-part-ii/ (Accessed 1 Mar 2018)
Snowden D (2016) SenseMaker ${ }^{\oplus}$, Cognitive Edge. Available at http://cognitive-edge. com/sensemaker/ (Accessed 20 Dec 2016)

Stige B et al. (2013) Where music helps: community music therapy in action and reflection. Ashgate, Farnham, UK; Burlington, VT

Tarr B, Launay J, Dunbar R (2014) Music and social bonding: "self-other" merging and neurohormonal mechanisms, Front Psychol 5(1096). https://doi.org/ 10.3389/fpsyg.2014.01096

Turino T (2008) Music as social life: the politics of participation. University of Chicago Press, Chicago

Turner E (2012) Communitas: the anthropology of collective joy. Palgrave Macmillan, New York

Västfjäll D, Juslin PN, Hartig T (2013) Music, subjective wellbeing, and health: the role of everyday emotions. In: Macdonald R, Kreutz G, Mitchell L (eds) Music, health and wellbeing. Oxford University Press, Oxford

Vickhoff B et al. (2013) Music structure determines heart rate variability of singers. Front Psychol 4(334). Available at http://journal.frontiersin.org/Journal/ 10.3389/fpsyg.2013.00334/full

Welch G et al. (2014) Singing and Social Inclusion, Front Psychol 5(803). https:// doi.org/10.3389/fpsyg.2014.00803

Whiten A (2007) The place of "deep social mind" in the evolution of human nature. In: Pasternak C (ed) What makes us human? Oneworld Publications, Oxford, pp. 146-163

Williams E, Dingle GA, Clift S (2018) A systematic review of mental health and wellbeing outcomes of group singing for adults with a mental health condition Eur J Public Health 28(6):1035-1042. https://doi.org/10.1093/eurpub/cky115

\section{Acknowledgements}

We acknowledge the generous support of the UK Arts and Humanities Research Council for the project 'The Hills Are Alive: combining the benefits of natural environments and group singing through immersive experiences' (AH/R009139/1) and the National Trust for their funding of the Fellowship of Hill and Wind and Sunshine project. Special thanks to Angie Power for proof-reading early drafts of this paper. We should also like to thank the singers who participated in this research for their time and for sharing their experiences with us.

\section{Competing interests}

The authors declare no competing interests.

\section{Ethical approval}

Ethical approval for 'The Hills Are Alive: combining the benefits of natural environments and group singing through immersive experiences' project was given by the University of York Physical Sciences Ethics Committee.

\section{Informed consent}

We confirm that informed consent was obtained from all participants and/or their legal guardians prior to participation, and participants were free to withdraw from the study at any time without explanation.

\section{Additional information}

Correspondence and requests for materials should be addressed to D.A.C.

Reprints and permission information is available at http://www.nature.com/reprints

Publisher's note Springer Nature remains neutral with regard to jurisdictional claims in published maps and institutional affiliations.

Open Access This article is licensed under a Creative Commons Attribution 4.0 International License, which permits use, sharing, adaptation, distribution and reproduction in any medium or format, as long as you give appropriate credit to the original author(s) and the source, provide a link to the Creative Commons license, and indicate if changes were made. The images or other third party material in this article are included in the article's Creative Commons license, unless indicated otherwise in a credit line to the material. If material is not included in the article's Creative Commons license and your intended use is not permitted by statutory regulation or exceeds the permitted use, you will need to obtain permission directly from the copyright holder. To view a copy of this license, visit http://creativecommons.org/ licenses/by/4.0/.

(C) The Author(s) 2020 